\title{
Laparoscopic management of choledochal cyst: T-tube or no T-tube
}

\author{
Manash Ranjan Sahoo*, Saurabh Kumar, and Ahmmed Shameel \\ Department of General Surgery, Sriram Chandra Bhanj Medical College, Cuttack, Odisha, India
}

\section{Introduction}

Laparoscopic management of choledochal cyst has been proved to be safe and feasible procedure. Choledochal cyst excision and hepaticojejunostomy has become standard operative management of type I and type II choledochal cyst. But anastomosis leak has been a significant problem in hepaticojejunostomy. Many precautionary measures have have been described in literature. Here we present a series to study usefulness of T-tube in reducing leak from hepaticojejunostomy in laparoscopic management of choledochal cyst.

\section{Materials and methods}

In this retrospective study of 18 patients including both male and female of age ranging from 17 to 32 years from June 2003 to February 2013. Patients presented with abdominal pain, jaundice, cholangitis or pancreatitis. All patients were investigated with routine blood tests, LFT, ultrasound, CT Scan and MRCP. Size of cyst ranged from $1.2 \mathrm{~cm}$ to $3 \mathrm{~cm}$. Type $1 \mathrm{a}, 1 \mathrm{~b}, 1 \mathrm{c}$ and type 2 were present in this study. Preanesthetic checkup was done and fit patients were put for surgery. Patients were subjected to laparoscopic choledochal cyst excision and hepaticojejunostomy, of which $10(60 \%)$ patients were given T-tube across the anastomosis site and $8(40 \%)$ patients were not given T-tube.

\section{Technique}

Under general anesthesia, the patients were placed in the reverse Trendelenburg position with abduction of both lower limbs. Pneumoperitoneum was established using veress needle.Port positions were-A $10 \mathrm{~mm}$ telescopic umbilical port, one $12 \mathrm{~mm}$ port put in left iliac fossa $5 \mathrm{~cm}$ lateral to umbilical port, one $5 \mathrm{~mm}$ port put in right hypochondrium in mid clavicular line and one $5 \mathrm{~mm}$ port in anterior axillary line in right hypochondrium. The cyst identified (Figure 1) and the anterior wall of the choledochal cyst was opened and all calculi removed. The cyst was transected $0.5-1.0 \mathrm{~cm}$ beneath the normal common hepatic duct. The cyst was dissected off the portal vein (Figure 2) and the entire cyst was resected. $15 \mathrm{~cm}$ jejunal limb was divided distal to the ligament of Treitz, using an Endo-GIA linear stapler. The mobilized Roux limb of the jejunum was pulled to the hepatic hilum in a retrocolic manner and a hepaticojejunostomy was created in an endto-side fashion by using interrupted suture using 3-0 vicryl (Figure 3). Cholecystectomy was done and a side-to-side jejunojejunostomy was then created at a level $50 \mathrm{~cm}$ distal to the hepaticojejunostomy, using the Endo-GIA linear stapler. A 16 Fr or 18 Fr T-tube was placed across the hepaticojejunostomy anastomosis to reach bifurcation of hepatic duct through the jejunum $10 \mathrm{~cm}$ from anastomosis in 10 patients (Figure 4) and in remaining 8 patients T-tube was not given. The skin ports were closed after the placement of an ADK drain in the subhepatic space.

\section{Results}

Post op period- Patients were started oral liquids on third day and gradually solid food was resumed. Drain was removed on $6^{\text {th }}$ day. Sutures were removed on third day. Patients were discharged on $7^{\text {th }}$ to $9^{\text {th }}$ day. Anastomosis leak occurred in two patients on $5^{\text {th }}$ and $6^{\text {th }}$ day which was identified first by clinical examination in the form of increased abdominal tenderness and increase in drain output and confirmed by ultrasound. Both patients were successfully managed by laparoscopic toileting and intra-corporeal suturing of leaked part of hepaticojejunostomy with non-absorbable suture. Both patients recovered fully and their post op period was uneventful. In all patients, follow up ultrasound and CT Scan was done 3 months, 6 months, 12 months, 18 months and 24 months.

Duration of surgery was $196.6 \pm 10.4$ minutes in T-tube group and $180 \pm 8.8 \mathrm{~min}$ in non T-tube group. Mean hospital stay was 8 (range: 7-10) days. There was postoperative complication in the form of anastomosis leak that occurred in two patients in non T-tube group. There was no leak in the T-tube group. There were no recurrences.

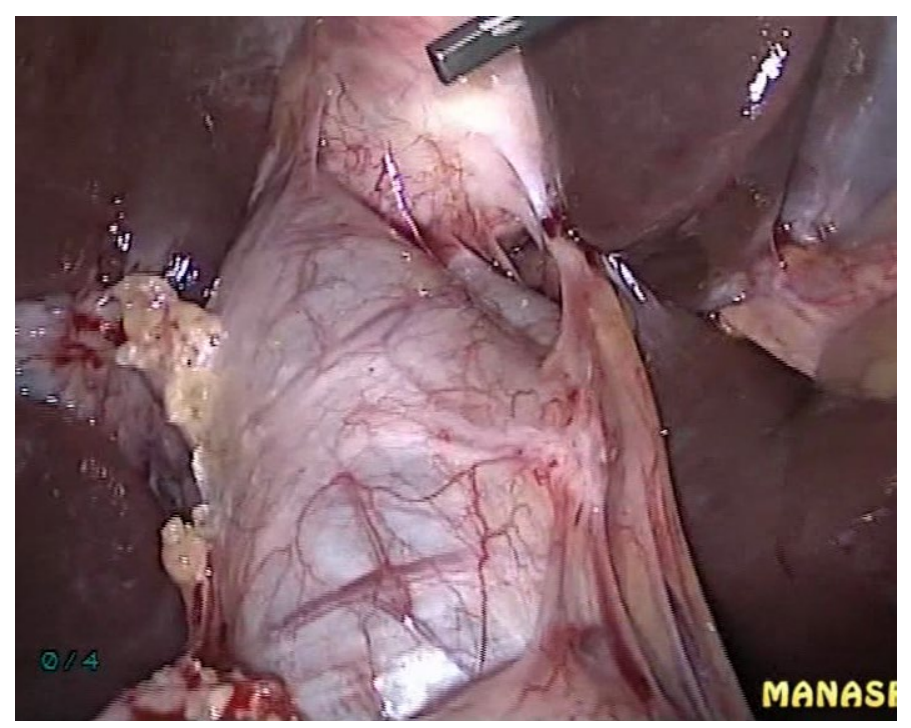

Figure 1. Choledochal cyst being seen.

Correspondence to: Dr. Manash Ranjan Sahoo, Department of General Surgery, Sriram Chandra Bhanj Medical College, Cuttack, Odisha, India, Tel: 9937025779 ; E-mail: manash67@gmail.com

Received: July 09, 2015; Accepted: August 10, 2015; Published: August 13, 2015 


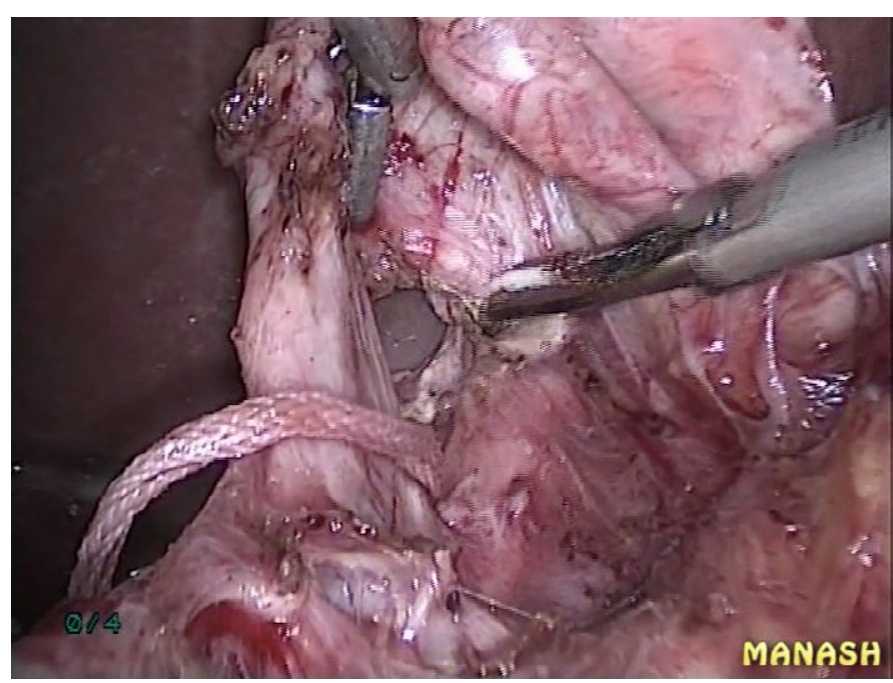

Figure 2. Cyst dissected off the portal vein.

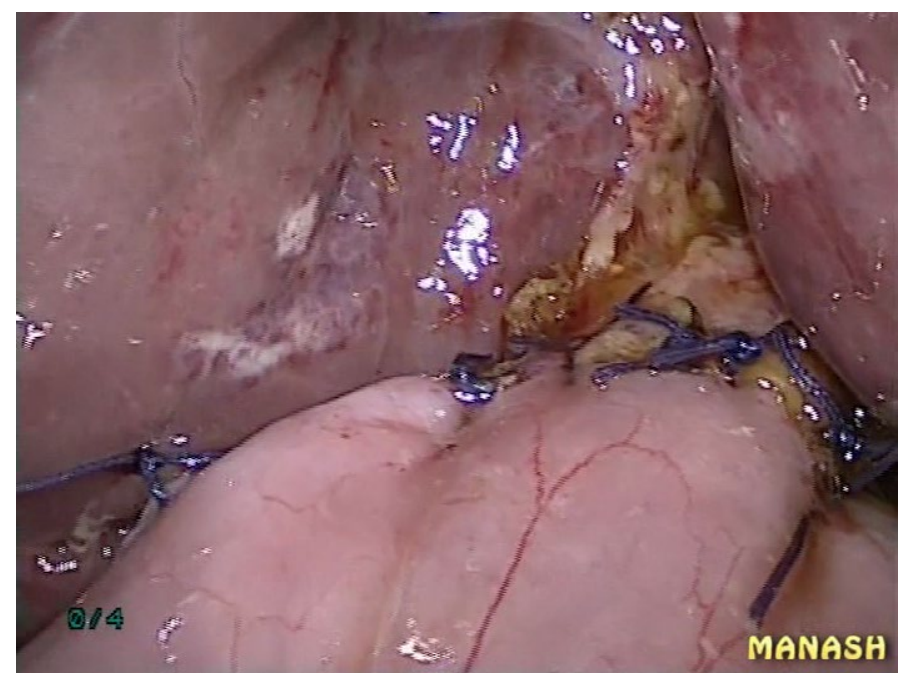

Figure 3. Completed hepaticojejunostomy.

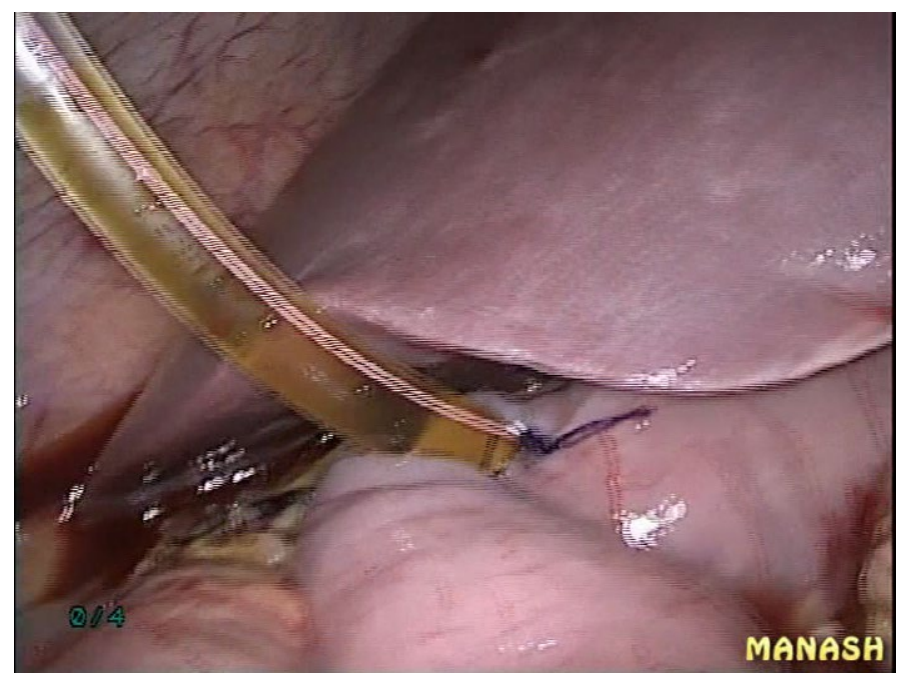

Figure 4. T-tube given across the hepaticojejunostomy as a stent.
Morbidity was greater in non T-tube group as leak occurred in two patients.

\section{Discussion}

Choledochal cyst is intra-hepatic or extra-hepatic dilatation ofbiliary tree. It is most common in children. The first clinical description of a patient with choledochal cyst was in 1852 by Douglas [1]. Choledochal cyst is most common in Asian population of Japan and China [2]. It is more common in females. Pediatric patients most commonly present as mass per abdomen, jaundice and right upper quadrant pain [3]. This triad is present in only $10 \%$ of pediatric population. While adults many a times present with complications like pancreatitis, cholangitis, biliary cirrhosis, cholangiocarcinoma etc. Most commonly used classification is Todani modification of Alonso-Lej classification. Diagnosis is done by LFT, ultrasound, CT Scan, ERCP and MRCP. Treatment include excision of cyst and hepaticojejunostomy for type 1,2 and 4b. Excision of cyst and hepaticojejunostomy can be done with open or laparoscopic method. Laparoscopic management of choledochal cyst has become standard treatment option. It achieves results with minimum post-op pain and morbidity. In laparoscopic hepaticojejunostomy, anastomosis leak and stricture are two very bothersome complication. Stricture can be present in as many as $73 \%$ of cases [4]. Stricture can be managed by percutaneous methods or revision of surgery. Important causes of leak includes interruption of blood supply to the remaining portion of hepatic duct and pressure on anastomosis in post op period. Proper blood supply in remaining hepatic duct can be achieved by meticulous dissection of hepatic duct and choledochal cyst and using scissors instead of cautery. The second cause of anatomosis leak can be avoided by using T-tube across the hepaticojejunostomy to reach the hepatic hilum. As T-tube maintains the bile flow giving time to the anastomosis to heal. In our study, T-tube group had no leak in comparison to non T-tube group, 2 anastomosis leak occurred.

\section{Conclusion}

We conclude that T-tube has a role in prevention of anastomosis leak in hepaticojejunostomy in laparoscopic management of choledochal cyst.

\section{References}

1. Douglas AH (1852) Case of dilatation of common bile duct. Monthly J Med Sci 14: 97.

2. Hung MH, Lin LH, Chen DF, Huang CS (2011) Choledochal cysts in infants and children: experiences over a 20-year period at a single institution. Eur J Pediatr 170: 1179-1185. [Crossref]

3. Miyano T, Yamataka A, Kato Y, Segawa O, Lane G, et al. (1996) Hepaticoenterostomy after excision of choledochal cyst in children: a 30 -year experience with 180 cases. $J$ Pediatr Surg 31: 1417-1421. [Crossref]

4. Stain SC, Guthrie CR, Yellin AE, Donovan AJ (1995) Choledochal cyst in the adult Ann Surg 222: 128-133. [Crossref]

Copyright: (C2015 Sahoo MR. This is an open-access article distributed under the terms of the Creative Commons Attribution License, which permits unrestricted use, distribution, and reproduction in any medium, provided the original author and source are credited. 